\title{
Transient uniocular visual loss on deviation of the eye in association with intraorbital tumours
}

\author{
P G BRADBURY,* I S LEVY, $†$ W I McDONALD* \\ From the National Hospital for Nervous Diseases*, Queen Square, and Moorfields Eye Hospital, $†$ London, UK
}

SUMMARY Five patients with unilateral orbital tumours are described in whom transient loss of vision occurred on deviation of the affected eye from the primary position. Other presenting features were diplopia, proptosis, poor visual acuity, visual field defects, pupillary abnormalities, fundal changes and altered colour vision. Abnormalities on fluorescein angiography suggest that the visual loss is due to transient ischaemia. Temporary uniocular loss of vision on eye movement may be an early sign of an intra-orbital mass.

The usual presentation of orbital tumours is that of progressive visual failure associated with proptosis. ${ }^{12}$ Rarely patients may give a history of transient obscurations of vision in the affected eye associated with movement from the primary position. This symptom may be associated with transient diplopia experienced after initial movement and shortly before loss of vision in the affected eye. There may be a significant delay of the order of 5 to 10 seconds before the visual loss occurs.

We present the case histories and findings in five such patients.

\section{Case reports}

Case 1 A 37 year old male lorry driver presented with a 7 week history of transient loss of vision in the right eye on gaze to the right. This symptom began after a minor road traffic accident in which he sustained a whiplash injury to the neck. Over the same 7 week period he experienced rightsided occipital headache. He first noticed visual disturbance when, whilst reversing his lorry with his head turned to the right, he went momentarily blind in the right eye. His vision returned after approximately 15 seconds on returning his gaze straight ahead. He subsequently noticed this symptom each time he turned his head to the right. He also developed double vision on right gaze. On examination, there was no proptosis. The visual acuity was $6 / 6$ bilaterally uncorrected. The blind spot was enlarged on the right and the optic disc was swollen (fig 1). On gaze to the right, after approximately

Address for reprint requests: Dr P Bradbury, National Hospital for Nervous Diseases, Queen Square, London WCIN 3BG, UK.

Received 31 January 1986 and in revised form 4 June 1986. Accepted 15 June 1986
5 seconds, he lost vision in the right eye, the pupil becoming dilated and fixed. On returning to the primary position, his sight and pupillary reactions returned. It was of interest to note that in the abducted position, the consensual reflex was abolished in the right eye. The intraocular pressures in the primary position were $17 \mathrm{~mm} \mathrm{Hg}$ on the right and $19 \mathrm{~mm} \mathrm{Hg}$ on the left. On elevation and dextroversion the pressure in the right eye rose to 32 and $36 \mathrm{~mm} \mathrm{Hg}$ respectively. A fluorescein angiogram in the primary position (Prof A Bird and Mr M Saunders; fig 2a) showed changes attributable to swelling and ischaemia of the optic nerve head and surrounding choroid on the temporal side. The retinal arterioles were well perfused. With the eye abducted, the fluorescein angiogram (fig $2 \mathrm{~b}$ ) showed almost no perfusion of the optic nerve head, the surrounding choroid or retinal arterioles.

Plain radiographs of the skull and optic foramina showed no abnormality, except gross opacification of the ethmoid air cells. The right optic nerve was explored through a lateral orbitotomy (Mr John Wright) and was found to be diffusely enlarged but of normal size at the orbital apex. An elipse of tissue was removed from the optic nerve sheath, the pathological appearances proving to be of diffuse meningioma. The patient's obscurations of vision and diplopia stopped postoperatively, but he was lost to follow-up shortly afterwards. Case 2 A 37 year old male sales agent presented with a history that 11 months previously he had experienced "fairly severe" left frontal and periocular pain lasting for 3 days. One week later, he noticed that the vision in the left eye seemed somewhat distorted on gaze to the left. Two months later this distortion seemed worse and he noticed diplopia on left gaze. By the time of presentation he complained that on sustained gaze to the left, the vision in the left eye "blacked out" with associated "discomfort" round the left eye, both symptoms disappearing when he looked to the front again. On examination, there was an extra-axial proptosis of $4 \mathrm{~mm}$ on the left. Visual acuities were $6 / 5$ on the right and $6 / 12$ on the left, both uncorrected. The visual fields were normal. Fundoscopy revealed left optic disc swelling with venous 


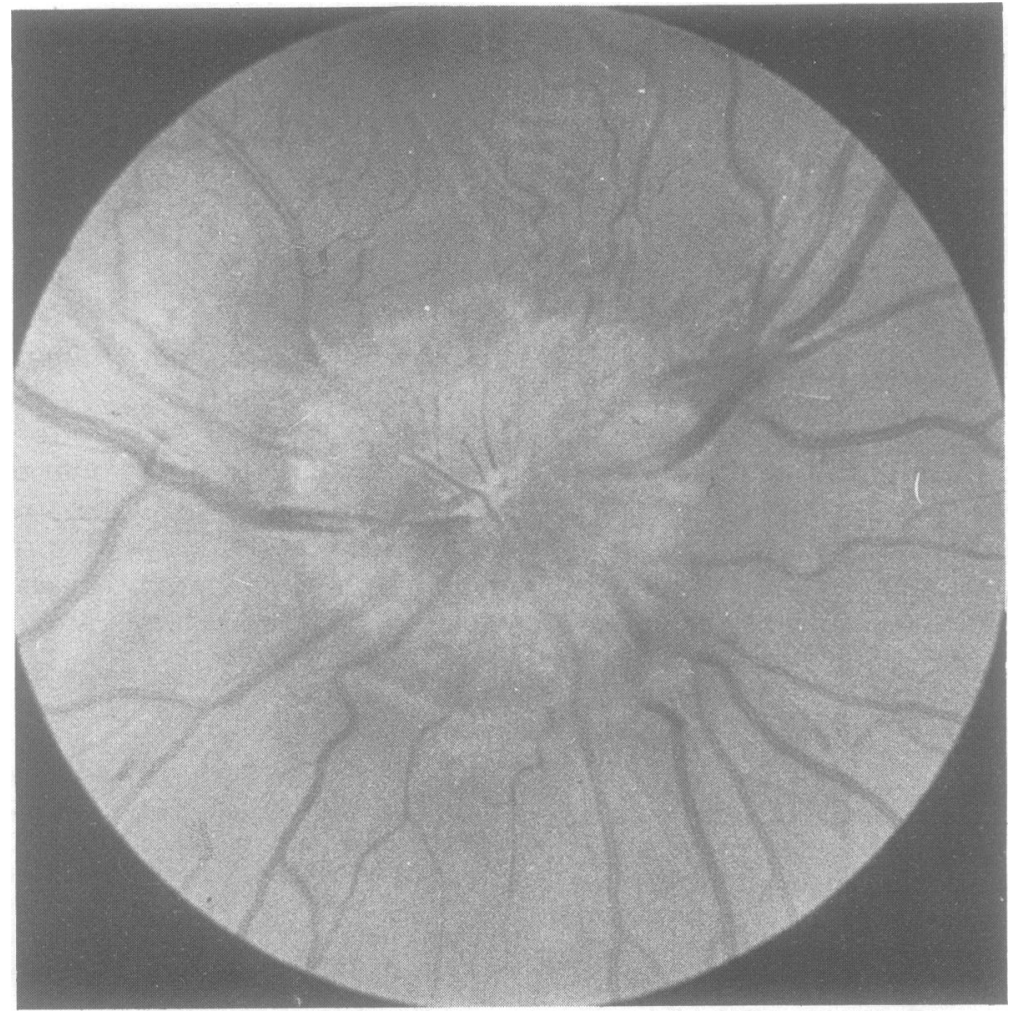

Fig 1 Swollen right optic disc in case 1

engorgement and choroidal folds running through the macular area. In the primary position, there was anisocoria, the left pupil being larger than the right. On laevoversion, after 10 seconds, the patient lost vision in the left eye. A relative afferent pupillary defect was noted in the left eye on left gaze which disappeared on regaining the primary position. The intraocular pressures were $11 \mathrm{~mm} \mathrm{Hg}$ on the right and $12 \mathrm{~mm} \mathrm{Hg}$ on the left, rising on upgaze to $13 \mathrm{~mm} \mathrm{Hg}$ and $18 \mathrm{~mm} \mathrm{Hg}$ respectively. Plain radiographs of the orbit were normal. CT scan revealed a large cigar-shaped mass in the left orbit indistinguishable from the medial rectus muscle (fig 3). A lateral orbitotomy was performed (Mr John Wright), which revealed a large pale tumour in the medial rectus muscle. This was shelled out and was found histologically to be a granular cell myoblastoma arising within the muscle. Postoperatively the patient's vision returned to normal.

Case 3 A 26 year old female secretary presented with a history that 5 months previously she had developed pain in the left eye on movement. Two months later she noticed some blurring of vision in that eye which remained relatively static until it deteriorated further 3 weeks prior to presentation. Five weeks before being seen, she noticed diplopia on extreme gaze to the left. Two weeks later, she began to notice that on standing up quickly she would transiently lose vision in the left eye. Shortly after admission to hospital for investigation, she began to notice that, on gaze to the left, the vision in the left eye would be lost spontane- ously, improving on return to the primary position. On clinical examination, there was no proptosis. Her visual acuities were $6 / 6$ on the right and $6 / 9$ on the left corrected. The blind spot was enlarged on the left. Colour vision was normal on the right and markedly impaired on the left. Fundoscopy revealed diffuse greyish swelling of the left optic disc. There was a relative afferent pupillary defect on the left. The left pupil dilated on elevation. The patient complained of diplopia on gaze to the left and lost vision in the left eye after approximately 10 seconds of sustained laevoversion. Her vision returned on regaining the primary position. The intraocular pressure was $12 \mathrm{~mm} \mathrm{Hg}$ bilaterally, the pressure in the left eye rising to $24 \mathrm{~mm} \mathrm{Hg}$ on upgaze. Plain radiographs of the orbits were normal. CT scan revealed a diffusely swollen left optic nerve (fig 4). The VEPs (Dr AM Halliday) obtained from the right eye were normal, but whole field stimulation of the left eye produced a small positive response at $125 \mathrm{~ms}$ and a large negative response on all the recordings at $159 \mathrm{~ms}$. The patient's symptoms remained static until 8 months later when her visual acuity deteriorated rapidly to $3 / 60$. The patient underwent a left fronto-orbital exploration (Prof Lindsay Symon) and a left optic nerve glioma was removed. The patient has remained well since with normal vision in the right eye.

Case 4 A 36 year old female civil servant presented with a history that 6 months previously she had begun to notice that, on turning her eyes to the right, after approximately 5 

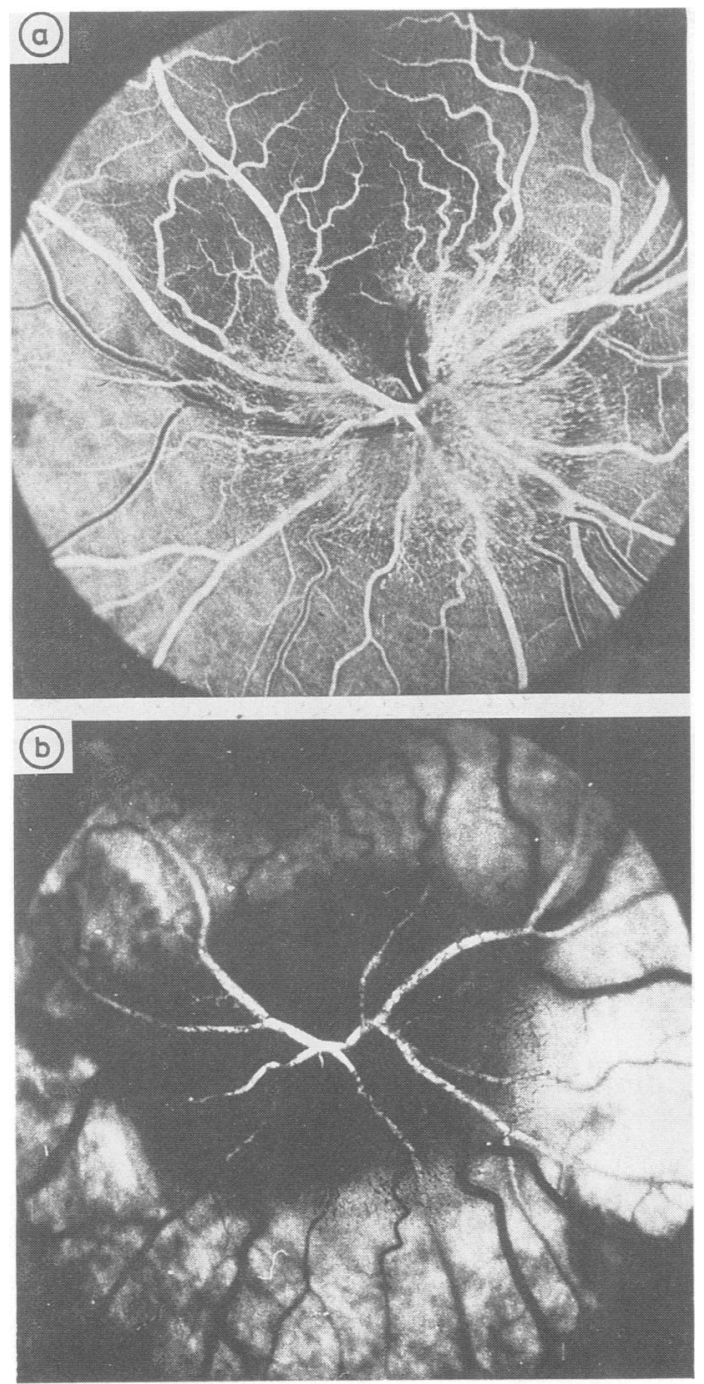

Fig 2a Fluorescein angiogram of right eye in primary position

Fig 2b Case 1 Fluorescein angiogram of right eye abducted

seconds she would lose the vision in the right eye. Her sight would return 10 or 20 seconds after looking straight ahead again. Two months later she noticed that shortly before losing vision in the right eye on gaze to the right, she would develop diplopia with horizontal separation of the images. These symptoms had slowly worsened prior to her admission to hospital. On examination, her visual acuities were $6 / 5$ on the left and 6/9 on the right. Fundoscopy revealed a swollen right optic disc. One month later she was noticed to have a $4 \mathrm{~mm}$ axial proptosis on the right. There was a right relative afferent pupillary defect and on dextroversion the right pupil dilated. Vision in the right eye was lost approximately 8 seconds after ocular movement. A fluorescein angiogram

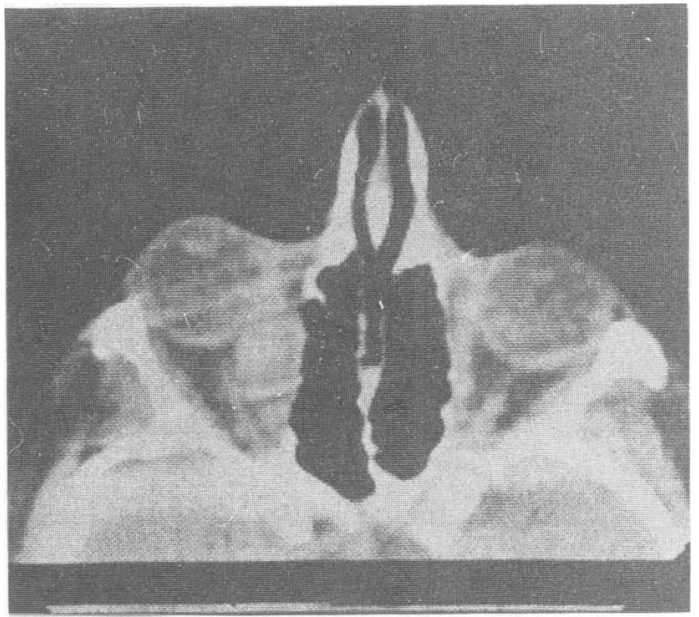

Fig 3 Case 2 CT scan showing mass in medial rectus on left

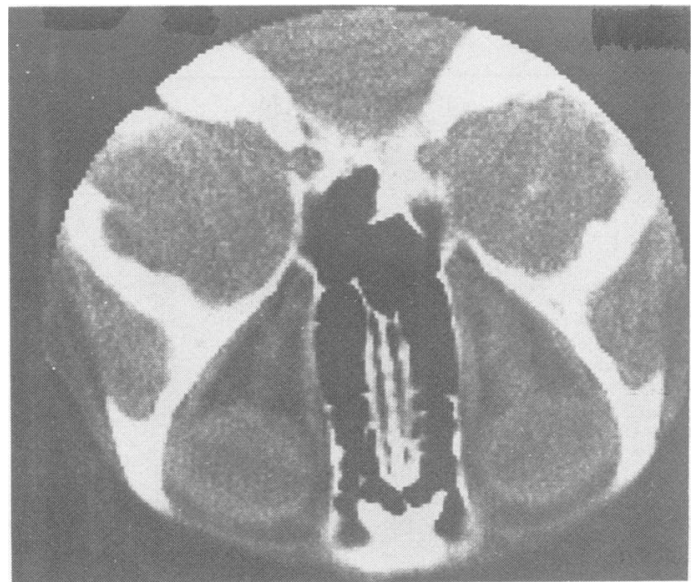

Fig 4 Case 3 CT scan showing diffusely swollen left optic nerve

showed early leakage of dye from the right disc. An orbital CT scan at that time was reported as normal. Two months later the visual acuity on the right had deteriorated to $6 / 18$ with reduced colour vision. There was limitation of dextroversion and elevation on the right. A further CT scan revealed a swollen right optic nerve, the appearances being suggestive of an optic nerve sheath meningioma (fig 5). Her VEPs (Prof Hisaka Ikeda) were normal on the left, the pattern evoked responses from the right eye being subnormal and delayed $(130 \mathrm{~ms})$. Light rise in the electro-oculogram (Prof Hisaka Ikeda), which provides a measure of the overall metabolic and functional integrity of the pigment epithelium and the photoreceptor outer segment, was normal on the left and slightly reduced on the right. ERGs recorded from the right eye using a contact lens electrode showed normal rod function with a normal ERG dark adaptation curve and normal adapted b-wave threshold. The cone mediated 


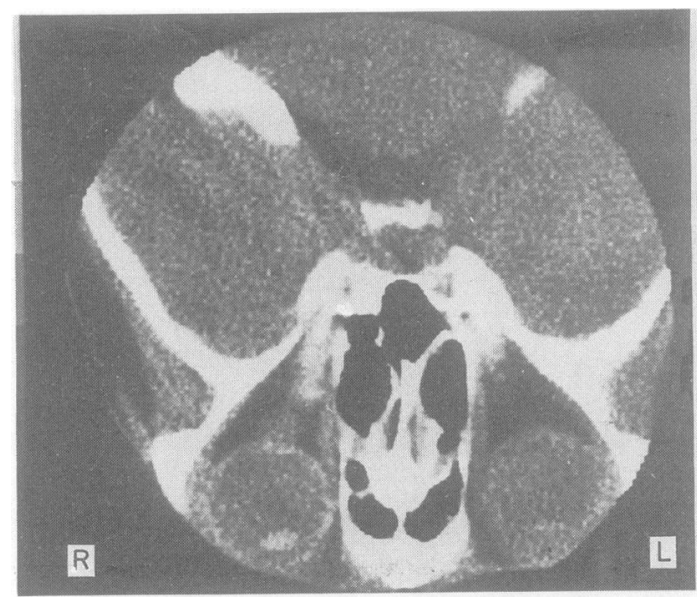

Fig 5 Case 4 CT scan showing swollen right optic nerve

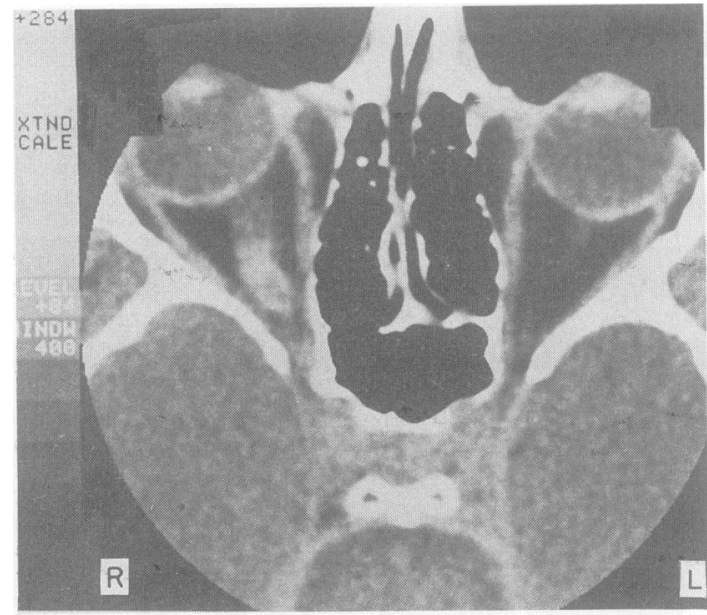

Fig 6 Case 5 CT scan showing thickened right optic nerve

Table Summary of clinical findings and investigations

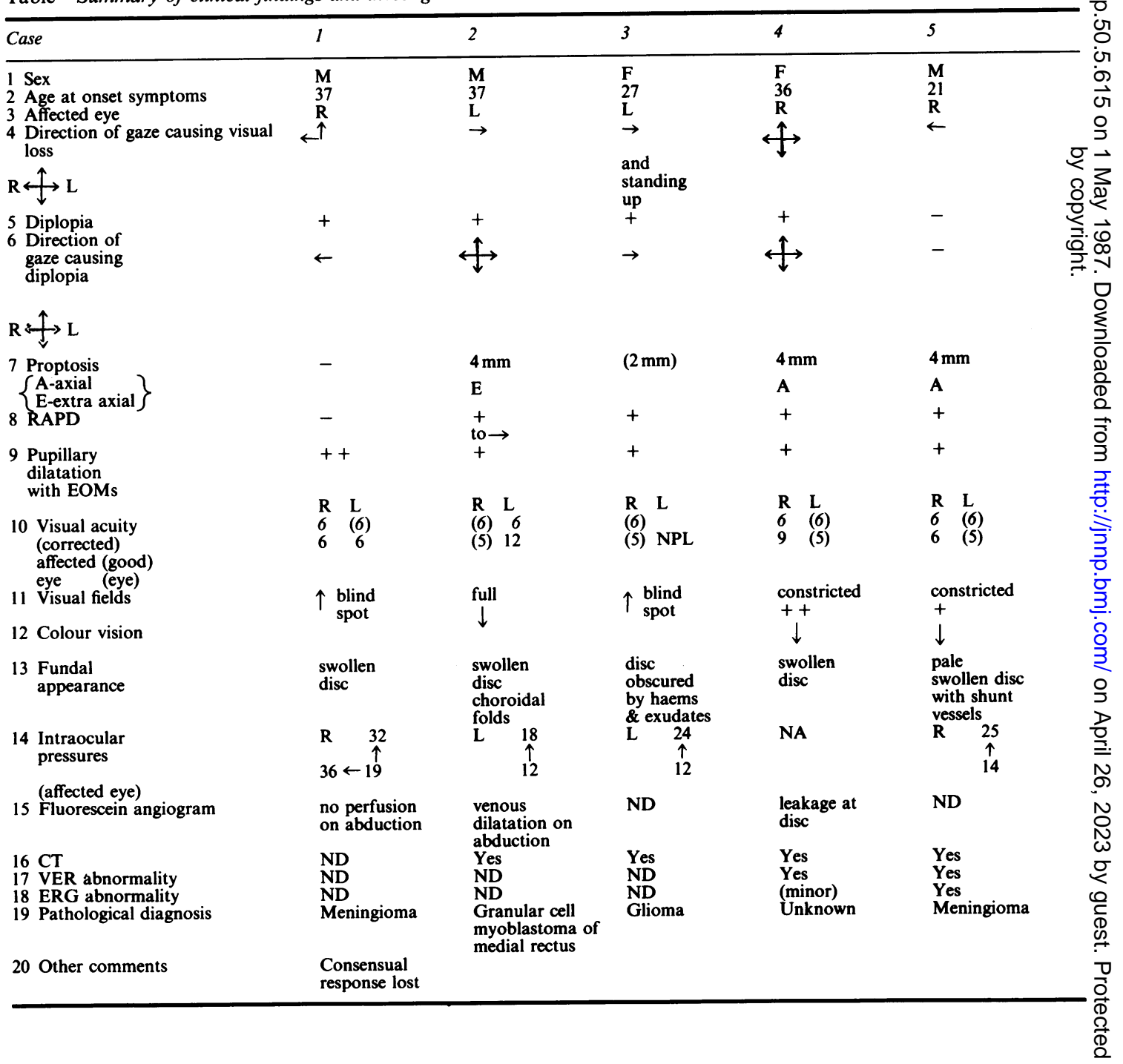


response was slightly subnormal. The averaged light adapted ERGs recorded using lateral canthi electrodes showed a small but significant interocular difference, the responses from the left eye being $20 \%$ larger than those from the right. The patient was reluctant to undergo surgery and has been managed conservatively, her vision gradually deteriorating in the affected eye.

Case 5 A 21 year old male engineering fitter presented with a history of slowly worsening mistiness of vision in the right eye over the previous 18 months. Three months prior to being seen, he began to experience occasional stabbing pains behind the right eye. On examination, there was an axial proptosis of $4 \mathrm{~mm}$ on the right. Visual acuities were $6 / 6$ on the right and $6 / 5$ on the left, both uncorrected. Colour vision was markedly reduced on the right. There was severe constriction of the right visual field. Fundoscopy showed a swollen right optic disc with associated large shunt vessels. In the primary position, the pupils were equal but with a right relative afferent pupillary defect. Movement of the right eye was limited on up and down gaze and on gaze to the right. On sustained gaze to the right, the patient lost vision in the right eye and right pupil dilated. Vision in the right eye returned approximately 10 seconds after regaining the primary position. The intraocular pressure was $14 \mathrm{~mm} \mathrm{Hg}$ bilaterally, rising to $25 \mathrm{~mm} \mathrm{Hg}$ on the right on elevation. Plain skull radiographs showed no abnormality. A CT scan demonstrated a widened right optic nerve (fig 6). VEPs (Prof Hisaka Ikeda) from the left eye were found to be normal. However, those from the right eye were subnormal, broad and slightly delayed $(160 \mathrm{~ms})$ on central gaze, but were completely abolished on gaze to the right, co-incident with the patient's reported loss of vision. No such "blackout phenomenon" could be demonstrated on ERG. The patient subsequently underwent a right fronto-orbital removal of an optic nerve sheath meningioma (Prof Symon).

\section{Discussion}

The clinical syndrome of progressive unilateral visual failure, with or without proptosis, may be produced by a wide range of intra-orbital disorders. In our patients three different intra-orbital tumours were found; optic nerve sheath meningioma, optic nerve glioma and medial rectus granular cell myoblastoma. ${ }^{3}$ The presentation of such cases with transient obscuration of vision in the affected eye with or without other symptoms or signs suggestive of an intraorbital lesion is uncommon, and such transient visual loss may not be reported by the patient despite being elicitable on clinical examination (Case 5). Furthermore, there was considerable variation in the clinical signs in our five cases when first seen (table).

The changes found on fluorescein angiography suggest that the transient loss of vision is secondary to temporary occlusion of the ophthalmic artery resulting from restriction of movement of the vessel in relation to the tumour. Such a mechanism of transient arterial occlusion resulting in optic nerve and/or retinal hypoperfusion would be compatible with the abolition of the VER seen in Case 5. Of particular interest is the loss of the consensual reflex in the abducted position in Case 1. It may be inferred that this efferent defect was due to transient ischaemia of the ciliary body. The preservation of the ERG at a time when the VEP was abolished in Case 5 suggests that ischaemia of the optic nerve was more important than retinal ischaemia in producing the visual loss in this case.

The authors are grateful to Mr John Wright and Dr GM Stern for their permission to report their patients, and to Miss Zeppetella for her secretarial assistance.

\section{References}

1 Jones IS, Jacobiac FA, eds. Diseases of the Orbit. New York: Harper and Row, 1979.

2 McDonald WI. The Symptomatology of Tumours of the Anterior Visual Pathways. Can J Neurol Sci 1982;9: 381.

3 Moriaty P, Garner A, Wright JE. Case report on granular cell myoblastoma arising within the medial rectus muscle. Br J Ophthalmol 1983;67:17-22. 